\title{
Student difficulties with finding the fine structure corrections to the energy spectrum of the hydrogen atom using degenerate perturbation theory
}

\author{
Chandralekha Singh, Emily Marshman, and Christof Keebaugh \\ Department of Physics and Astronomy, University of Pittsburgh, Pittsburgh, PA 15260
}

\begin{abstract}
We discuss an investigation of student difficulties with the fine structure corrections to the energy spectrum of the hydrogen atom in the context of degenerate perturbation theory (DPT). The investigation was carried out in advanced quantum mechanics courses by administering free-response and multiple-choice questions and conducting individual interviews with students. We discuss the common student difficulties related to these concepts. These findings provide guidelines for creating learning tools to help students develop a functional understanding of concepts involving the fine structure corrections to the energy spectrum of a hydrogen atom.
\end{abstract}

\section{INTRODUCTION AND BACKGROUND}

The hydrogen atom has played a significant role in the development of quantum mechanics (QM). Specifically, the discrete energy levels observed by the spectroscopists for the hydrogen atom led Bohr to propose his model with quantized energy levels. Further investigations showed that perturbations such as the fine structure term in the Hamiltonian cause shifts in the energy spectrum of the hydrogen atom. The fine structure of the hydrogen atom is the combined effect of the relativistic correction and the spin-orbit interaction since both components produce the same order of magnitude corrections to the energies of the hydrogen atom.

The Time-Independent Schrödinger Equation (TISE) for the hydrogen atom Hamiltonian with the fine structure corrections cannot be solved exactly. Nevertheless, since the fine structure term is significantly smaller than the unperturbed Hamiltonian, perturbation theory is an excellent method for determining the approximate solutions to the TISE for finding the corrections to the unperturbed energy spectrum of the hydrogen atom due to fine structure. Moreover, due to the degeneracy in the energy spectrum of the hydrogen atom, degenerate perturbation theory (DPT) must be used to find the perturbative corrections due to fine structure.

It is important to help students develop a functional understanding of DPT in order to find the fine structure corrections. However, QM is a challenging subject for upper-level undergraduate and graduate students in physics (e.g., see Refs. [118]) and students often struggle with DPT. Here, we discuss an investigation of student difficulties with finding the firstorder perturbative corrections to the energy spectrum of the hydrogen atom due to fine structure which can be helpful in developing curricula (e.g. Quantum Interactive Learning Tutorials (QuILTs)) to improve student understanding [9-11,14].

We first discuss the requisite knowledge students must have to use DPT in general and in the context of the fine structure corrections. Perturbation theory (PT) is a powerful approximation method for finding the energies and the energy eigenstates for a system for which the TISE is not exactly solvable. The Hamiltonian $\hat{H}$ for the system can be expressed as the sum of two terms, the unperturbed Hamiltonian $\hat{H}^{0}$ and the perturbation $\hat{H}^{\prime}$, i.e., $\hat{H}=\hat{H}^{0}+\hat{H}^{\prime}$. The TISE for the unperturbed Hamiltonian, $\hat{H}^{0} \psi_{n}^{0}=E_{n}^{0} \psi_{n}^{0}$, is exactly solvable where $\psi_{n}^{0}$ is the $n^{\text {th }}$ unperturbed energy eigenstate and
$E_{n}^{0}$ the unperturbed energy. PT builds on the solutions of the TISE for the unperturbed case. Using PT, the energies can be approximated as $E_{n}=E_{n}^{0}+E_{n}^{1}+E_{n}^{2}+\ldots$ where $E_{n}^{i}$ for $i=$ $1,2,3, \ldots$ are the $i^{\text {th }}$ order corrections to the $n^{\text {th }}$ energy of the system. We focus on the first-order perturbative corrections to the energies since they are usually the dominant corrections. In nondegenerate perturbation theory (NDPT), the first-order corrections to the energies are $E_{n}^{1}=\left\langle\psi_{n}^{0}\left|\hat{H}^{\prime}\right| \psi_{n}^{0}\right\rangle$, and the first-order corrections to the unperturbed energy eigenstates are $\left|\psi_{n}^{1}\right\rangle=\sum_{m \neq n} \frac{\left\langle\psi_{m}^{0}\left|\hat{H}^{\prime}\right| \psi_{n}^{0}\right\rangle}{\left(E_{n}^{0}-E_{m}^{0}\right)}\left|\psi_{m}^{0}\right\rangle$. In both the first-order corrections to the energies and the energy eigenstates, $\left\{\left|\psi_{n}^{0}\right\rangle\right\}$ is a complete set of eigenstates of $\hat{H}^{0}$. When the eigenvalue spectrum of $\hat{H}^{0}$ has degeneracy (two or more eigenstates of $\hat{H}^{0}$ have the same energy, i.e., two or more diagonal elements of $\hat{H}^{0}$ are equal when the eigenstates of $\hat{H}^{0}$ form the basis), the equations for the first order corrections to the energies and energy eigenstates from NDPT are still valid provided one uses a good basis. For a given $\hat{H}^{0}$ and $\hat{H}^{\prime}$, we define a good basis as consisting of a complete set of eigenstates of $\hat{H}^{0}$ that diagonalizes $\hat{H}^{\prime}$ in each degenerate subspace of $\hat{H}^{0}$ [19].

For a hydrogen atom, one can use DPT to find the perturbative corrections to the energies due to the fine structure term. Using standard notations, the Hamiltonian of a hydrogen atom with fine structure is $\hat{H}=\hat{H}^{0}+\hat{H}_{f s}^{\prime}$, in which the unperturbed Hamiltonian, $\hat{H}^{0}=\frac{\hat{p}^{2}}{2 m}-\frac{e^{2}}{4 \pi \epsilon_{0}}\left(\frac{1}{r}\right)$, accounts only for the interaction of the electron with the nucleus via Coulomb attraction. The solution of the TISE for the hydrogen atom with Coulomb potential energy gives the unpertubed energies $E_{n}^{0}=-\frac{13.6 \mathrm{eV}}{n^{2}}$, where $n$ is the principal quantum number. For each subspace corresponding to the principal quantum number $n$, the energy spectrum of $\hat{H}^{0}$ is $2 n^{2}$-fold degenerate. Using standard notations, the fine structure perturbation is $\hat{H}_{f s}^{\prime}=\hat{H}_{r}^{\prime}+\hat{H}_{S O}^{\prime}$ where $\hat{H}_{r}^{\prime}=-\frac{\hat{p}^{4}}{8 m^{3} c^{2}}$ is the relativistic correction term and $\hat{H}_{S O}^{\prime}=\left(\frac{e^{2}}{8 \pi \epsilon_{0}}\right) \frac{1}{m^{2} c^{2} r^{3}}(\vec{L} \cdot \vec{S})$ is the spinorbit interaction term. Since the relativistic correction and the spin-orbit terms are of the same order of magnitude, they are combined as the fine structure term $\hat{H}_{f s}^{\prime}$.

To find a good angular basis for the fine structure corrections to the energies of a hydrogen atom, one can diagonalize the fine structure perturbation $\hat{H}_{f s}^{\prime}$ in each degenerate subspace of the unperturbed Hamiltonian $\hat{H}^{0}$. The angular basis refers to the part of the basis that combines both the spin and 
orbital angular momenta. Since the focus is on each degenerate subspace of the unperturbed Hamiltonian $\hat{H}^{0}$ and whether $\hat{H}_{f s}^{\prime}$ is diagonal in each degenerate subspace of $\hat{H}^{0}$, we can restrict our discussion to one value of the principal quantum number $n$. Thus, in this entire discussion below, we will focus only on the $n=2$ degenerate subspace of $\hat{H}^{0}$. Also, in the questions that students were asked about the fine structure corrections to the energy spectrum of the hydrogen atom using the DPT, they were asked to fix the value of the principal quantum number to $n=2$.

We note that $\hat{H}^{0}$ for the hydrogen atom is spherically symmetric and the energy only depends on $n$. Thus, for $n=2$, $\hat{H}^{0}$ is diagonal when any complete set of orthogonal states is chosen for the angular part of the basis (or angular basis). Thus, so long as the radial part of the wavefunction $R_{n l}(r)$ (for a given principal quantum number $n$ and azimuthal quantum number $l$ ) corresponding to the eigenstates of $\hat{H}^{0}$ is chosen as the basis (which we will assume throughout here), the choice of a good basis for DPT amounts to choosing the angular basis appropriately for a given perturbation (ensuring that the perturbation matrix is a diagonal matrix in each degenerate subspace of $\hat{H}^{0}$ ). Thus, our focus here is on choosing a good angular basis.

Below, we consider each part of the perturbation $\hat{H}_{f s}^{\prime}=$ $\hat{H}_{r}^{\prime}+\hat{H}_{S O}^{\prime}$ separately and then together in order to reason about how to determine the angular part of a good basis. The operator $\hat{H}_{r}^{\prime}$ is spherically symmetric and the eigenvalues of $\hat{H}_{r}^{\prime}$ depend on quantum numbers $n$ and $l$. Thus, for both $\hat{H}^{0}$ and $\hat{H}_{r}^{\prime}$, for $n=2$ (which is the degenerate subspace of $\hat{H}^{0}$ we will focus on throughout our discussion), any complete set of orthogonal states can be chosen for the angular part of the basis so long as we take linear superposition of states with the same values of $l$. Under these conditions, any complete set of orthogonal states forms a good angular basis for finding the corrections to the energy of a hydrogen atom due to the relativistic correction term $\hat{H}_{r}^{\prime}$ only. On the other hand, since $\hat{H}_{S O}^{\prime}$ is proportional to $\vec{L} \cdot \vec{S}=\frac{1}{2}\left(\hat{J}^{2}-\hat{S}^{2}-\hat{L}^{2}\right)$, it is useful to note that the product states in the coupled representation $\left|l, j, m_{j}\right\rangle$ are eigenstates of the operator $\frac{1}{2}\left(\hat{J}^{2}-\hat{S}^{2}-\hat{L}^{2}\right)$ (in which all operators, e.g., $\hat{J}$, and the quantum numbers $l, j$ and $m_{j}$ are in standard notations and $\mathrm{s}=1 / 2$ has been suppressed from the states $\left|l, j, m_{j}\right\rangle$ since $\mathrm{s}=1 / 2$ is a fixed value for a hydrogen atom). Thus, an angular basis consisting of states in the coupled representation is a good angular basis for $\hat{H}^{0}$ and $\hat{H}_{S O}^{\prime}$ for DPT. Therefore, combining the relativistic and spin-orbit interaction contributions, a good angular basis for $\hat{H}^{0}$ and $\hat{H}_{f s}^{\prime}=\hat{H}_{r}^{\prime}+\hat{H}_{S O}^{\prime}$ is the coupled representation.

\section{METHODOLOGY}

Student difficulties with finding the corrections to the energy of the hydrogen atom due to fine structure using DPT were first investigated using four years of data involving responses to open-ended and multiple-choice questions administered after traditional instruction in relevant concepts to 52 upper-level undergraduates in a second-semester junior/senior level QM course and 42 first-year physics graduate students in the second-semester of the graduate core QM course. Additional insight was gained concerning these difficulties via responses of 13 students during a total of 45 hours of individual interviews in which they were asked to think aloud as they answered the questions posed without being disturbed. Only at the end, they were asked to clarify any issues.

In all of the questions asked in the investigation, students were given that the radial wavefunctions, $R_{n l}$, are always eigenstates of $\hat{H}^{0}$ and they must only focus on the angular part of the basis in order to find a good basis for DPT involving $\hat{H}^{0}$ and $\hat{H}_{f s}^{\prime}$. Additionally, in all questions, students were asked to consider only the $n=2$ subspace for which the unperturbed energy $E_{2}^{0}=-\frac{13.6 \mathrm{eV}}{4}$ is 8 -fold degenerate. We note that students were provided all relevant equations and information that were discussed in the introduction and background section and had learned about the coupled and uncoupled representations primarily via lecture-based instruction.

After analyzing responses of 32 undergraduates on similar questions administered in the two previous years, we posed the following question to 20 undergraduate and 42 graduate students in the following two years as part of an in-class quiz after traditional lecture-based instruction to examine student difficulties with the fine structure corrections to the energy spectrum of the hydrogen atom in the context of DPT (restricted to $n=2$ degenerate subspace of $\hat{H}^{0}$ ).

Q1. A perturbation $\hat{H}^{\prime}$ acts on a hydrogen atom with the unperturbed Hamiltonian $\hat{H}^{0}=-\frac{\hbar^{2}}{2 m} \nabla^{2}-\frac{e^{2}}{4 \pi \epsilon_{0}}\left(\frac{1}{r}\right)$. For the perturbation $\hat{H}^{\prime}$, circle $\mathbf{A} \mathbf{L} \mathbf{L}$ of the representations that can be chosen as the angular part of a good basis and explain your reasoning. Assume that for all cases, the principal quantum number is restricted to $n=2$.

i. Coupled representation

ii. Uncoupled representation

iii. any arbitrary orthonormal basis constructed with a linear combination of states in the coupled representation with same $l$

iv. any arbitrary orthonormal basis constructed with a linear combination of states in the uncoupled representation with same l

v. Neither coupled nor uncoupled representation.

In $\mathrm{Q} 1$, the perturbation operator $\hat{H}^{\prime}$ is a proxy for the operators $\hat{H}_{r}^{\prime}, \hat{H}_{S O}^{\prime}$, and $\hat{H}_{f s}^{\prime}$ listed individually in three separate questions. Since the unperturbed Hamiltonian $\hat{H}^{0}$ and the relativistic correction term $\hat{H}_{r}^{\prime}$ are diagonal in any angular basis consisting of a complete set of orthogonal states given in Q1, options i, ii, iii, and iv are all correct for a good angular basis for finding the perturbative corrections for the relativistic correction term $\hat{H}_{r}^{\prime}$ as a perturbation on $\hat{H}^{0}$ for the $n=2 \mathrm{de}$ generate subspace of $\hat{H}^{0}$. Also, since $\hat{H}^{0}$ and $\hat{H}_{S O}^{\prime}$ are both diagonal in the coupled representation, a good angular basis for finding perturbative correction for $\hat{H}_{S O}^{\prime}$ is given by option i only (coupled representation) in Q1. For the fine structure term, $\hat{H}_{f s}^{\prime}=\hat{H}_{r}^{\prime}+\hat{H}_{S O}^{\prime}$, a good angular basis for finding the 
TABLE I. The percentages of undergraduate $(\mathrm{U})(N=20)$ and graduate $(\mathrm{G})$ students $(N=42)$ who provided the correct response (chose all of the possible correct representations) in Q1.

\begin{tabular}{|c|c|c|c|}
\hline$\hat{H}^{\prime}$ Operator & Correct Answer & $\mathrm{U}(\%)$ & $\mathrm{G}(\%)$ \\
\hline$\hat{H}_{r}^{\prime}$ & $\mathrm{i}, \mathrm{ii}, \mathrm{iii}, \mathrm{iv}$ & 10 & 17 \\
\hline$\hat{H}_{S O}^{\prime}$ & $\mathrm{i}$ & 20 & 38 \\
\hline$\hat{H}_{f s}^{\prime}$ & $\mathrm{i}$ & 10 & 29 \\
\hline
\end{tabular}

TABLE II. The percentages of undergraduate (U) $(N=20)$ and graduate $(\mathrm{G})(N=42)$ students who did NOT choose states in the coupled representation for the listed perturbation $\hat{H}^{\prime}$ in Q1.

\begin{tabular}{|c|c|c|}
\hline$\hat{H}^{\prime}$ Operator & $\mathrm{U}(\%)$ & $\mathrm{G}(\%)$ \\
\hline$\hat{H}_{r}^{\prime}$ & 50 & 67 \\
\hline$\hat{H}_{S O}^{\prime}$ & 60 & 36 \\
\hline$\hat{H}_{f s}^{\prime}$ & 75 & 55 \\
\hline
\end{tabular}

perturbative corrections is also a basis consisting of states in the coupled representation. Thus, only option $\mathrm{i}$ is correct for the fine structure term $\hat{H}_{f s}^{\prime}$ in Q1.

\section{STUDENT DIFFICULTIES}

Before discussing student difficulties tied specifically to finding a good basis for the fine structure corrections to the energy spectrum, we note that many students had difficulties with DPT in general. For example, some students did not even realize that DPT (as opposed to NDPT) should be used in this context since the unperturbed Hamiltonian for the hydrogen atom is $2 n^{2}$-fold degenerate. Other students knew that they had to use DPT to find the corrections to the unperturbed wavefunctions (since the denominator "blows up" in the equation for the corrections to the wave functions $\left.\left|\psi_{n}^{1}\right\rangle=\sum_{m \neq n} \frac{\left\langle\psi_{m}^{0}\left|\hat{H}^{\prime}\right| \psi_{n}^{0}\right\rangle}{\left(E_{n}^{0}-E_{m}^{0}\right)}\left|\psi_{m}^{0}\right\rangle\right)$. However, they did not realize that DPT should also be used to find the first-order corrections to the energies. They incorrectly claimed that in the first-order corrections to the energies $\left(E_{n}^{1}=\left\langle\psi_{n}^{0}\left|\hat{H}^{\prime}\right| \psi_{n}^{0}\right\rangle\right)$, no terms "blow up" so it was appropriate to use NDPT (these students did not attempt to find a good basis for DPT when finding the fine structure corrections).

Moreover, even if students realized that DPT should be used for the fine structure corrections to the energies of the hydrogen atom, many of them admitted that they had memorized that the coupled representation is a good basis for finding the fine structure corrections. For example, one interviewed student stated, "I was always confused with coupled and uncoupled representation. I just memorized when to use which." Memorization of which basis to use often masked the fact that students did not have a functional understanding of DPT for the fine structure corrections to the energy spectrum.

In the context of the fine structure corrections, students struggled to determine a good angular basis for each perturbation $\hat{H}_{r}^{\prime}, \hat{H}_{S O}^{\prime}$, and $\hat{H}_{f s}^{\prime}$ provided in Q1 and found it challenging to determine if a perturbation is diagonal in the $n=2$ degenerate subspace of the unperturbed Hamiltonian.
The results are summarized in Tables I and II. We note that in Q1, it is important that students identify that the coupled representation forms a good angular basis for $\hat{H}_{r}^{\prime}$ and $\hat{H}_{S O}^{\prime}$ since the coupled representation is the only option given that forms a good angular basis for the fine structure perturbation $\hat{H}_{f s}^{\prime}=\hat{H}_{r}^{\prime}+\hat{H}_{S O}^{\prime}$ (this is the basis in which the perturbation $\hat{H}_{f s}^{\prime}$ is diagonal in the $n=2$ degenerate subspace of $\hat{H}^{0}$ ). However, Table II shows that many students did not choose the coupled representation as a good angular basis for finding the corrections to the energies due to the relativistic correction only, the spin-orbit interaction only, and the fine structure.

One difficulty that prevented students from choosing the representation that forms a good angular basis (e.g., in response to Q1) was focusing only on $\hat{H}^{0}$ and not on $\hat{H}^{\prime}$ when finding a good angular basis. In response to Q1, students with this type of difficulty focused on the bases that make $\hat{H}^{0}$ diagonal but ignored consideration of $\hat{H}^{\prime}$ when finding a good basis. In particular, they chose a good basis based only upon the representation that makes $\hat{H}^{0}$ diagonal without considering whether the operator $\hat{H}_{f s}^{\prime}$ was diagonal in each degenerate subspace of $\hat{H}^{0}$ in that basis. For example, in interviews, some students claimed that the uncoupled representation was a basis that would make the operator $\hat{H}^{0}$ diagonal, and they then incorrectly chose the uncoupled representation as a good angular basis for the fine structure corrections. In their explanations, they correctly noted that states in the uncoupled representation were eigenstates of $\hat{H}^{0}$, but they did not consider the fact that $\hat{H}_{f s}^{\prime}$ is not diagonal in each degenerate subspace of $\hat{H}^{0}$ when states in the uncoupled representation are chosen as the angular basis. For example, in the $n=2$ subspace, the $\hat{H}_{f s}^{\prime}$ matrix is given below when the basis states are chosen in the uncoupled representation as $\left|l, m_{l}, m_{s}\right\rangle$ (the notations are standard and quantum numbers $m_{l}$ and $m_{s}$ correspond to the $\mathrm{z}$ component of the orbital and spin angular momenta, respectively) in the order $\left|0,0, \frac{1}{2}\right\rangle,\left|0,0,-\frac{1}{2}\right\rangle,\left|1,1, \frac{1}{2}\right\rangle,\left|1,1,-\frac{1}{2}\right\rangle,\left|1,0, \frac{1}{2}\right\rangle,\left|1,0,-\frac{1}{2}\right\rangle$, $\left|1,-1, \frac{1}{2}\right\rangle$, and $\left|1,-1,-\frac{1}{2}\right\rangle$ (where $\alpha$ is the fine structure constant):

$$
\hat{H}_{f s}^{\prime}=\frac{(-13.6 \mathrm{eV}) \alpha^{2}}{192}\left[\begin{array}{cccccccc}
15 & 0 & 0 & 0 & 0 & 0 & 0 & 0 \\
0 & 15 & 0 & 0 & 0 & 0 & 0 & 0 \\
0 & 0 & 3 & 0 & 0 & 0 & 0 & 0 \\
0 & 0 & 0 & 11 & 4 \sqrt{2} & 0 & 0 & 0 \\
0 & 0 & 0 & 4 \sqrt{2} & 7 & 0 & 0 & 0 \\
0 & 0 & 0 & 0 & 0 & 7 & 4 \sqrt{2} & 0 \\
0 & 0 & 0 & 0 & 0 & 4 \sqrt{2} & 11 & 0 \\
0 & 0 & 0 & 0 & 0 & 0 & 0 & 3
\end{array}\right] .
$$

Since many students only focused on the representation in which $\hat{H}^{0}$ was diagonal to determine a good basis, in the interviews, students were explicitly asked about the role of the perturbation term in the Hamiltonian in the choice of a good basis. They were asked to calculate some of the off-diagonal matrix elements of the $\hat{H}_{f s}^{\prime}$ matrix or at least reason conceptually about whether some of the off-diagonal matrix elements of $\hat{H}_{f s}^{\prime}$ would be non-zero in the uncoupled representation in 
the $n=2$ degenerate subpsace of $\hat{H}^{0}$ (see the preceding matrix). We find that the interviewed students often struggled to calculate or reason conceptually that the fine structure perturbation $\hat{H}_{f s}^{\prime}$ shown in the preceding matrix is not diagonal in the $n=2$ degenerate subspace of $\hat{H}^{0}$ in the uncoupled representation and that the uncoupled representation cannot be a good angular basis for finding the fine structure corrections.

\section{DEVELOPMENT OF A QUILT}

We developed and validated a Quantum Interactive Learning Tutorial (QuILT) which is being implemented in quantum mechanics classes. The QuILT strives to help students with the fine structure corrections to the energy spectrum of the hydrogen atom using degenerate perturbation theory. It uses an inquiry-based approach to learning and actively engages students in the learning process. It includes a pretest to be administered in class after traditional instruction in DPT and posttest to be administered after students have engaged with the DPT QuILT. The development of the QuILT started with an investigation of student difficulties via open-ended and multiplechoice questions administered after traditional instruction to advanced undergraduate and graduate students and conducting a cognitive task analysis from an expert perspective of the requisite knowledge. The QuILT strives to help students build on their prior knowledge and addresses common difficulties found via research, some of which were discussed in the previous section. The development of the QuILT went through a cyclic, iterative process. The preliminary version was developed based upon the task analysis and knowledge of common student difficulties. Next, the QuILT underwent many iterations among the three researchers and then was iterated several times with three physics faculty members to ensure that they agreed with the content and wording. It was also administered to graduate and advanced undergraduate students in individual think-aloud interviews to ensure that the guided approach was effective, the questions were unambiguously interpreted, and to better understand the rationale for student responses. During these semi-structured interviews, students were asked to think aloud while answering the questions. Students first read the questions on their own and answered them without interruptions except that they were prompted to think aloud if they were quiet for a long time. After students had finished answering a particular question to the best of their ability, they were asked to further clarify and elaborate on issues that they had not clearly addressed earlier. The next step involved evaluating the QuILT's impact on student learning and determining if the difficulties remained. Finally, modifications and improvements were made based upon the student and faculty feedback before administering the QuILT to students in various courses.

Students engage with the QuILT in small groups in class or as a guide for class discussions (or alone when using it as a self-paced learning tool in homework), and then they are administered a posttest in class. As students work through the QuILT, they are asked to predict what should happen in a given situation. Then, the QuILT strives to provide scaffolding and feedback as needed to bridge the gap between their initial knowledge and the level of understanding that is desired. Students are also provided checkpoints to reflect upon what they have learned and to make explicit the connections between what they are learning and their prior knowledge. They are given an opportunity to reconcile differences between their predictions and the guidance provided in the checkpoints before proceeding further. The QuILT uses a blend of qualitative and quantitative reasoning to improve students' understanding. For example, the QuILT requires qualitative understanding while responding to the hypothetical conversations and quantitative reasoning to determine the matrix elements of the operators $\hat{H}_{S O}^{\prime}$ and $\hat{H}_{r}^{\prime}$ in the coupled and uncoupled representations. Students verify statements in hypothetical conversations via quantitative reasoning.

\section{CONCLUSION AND FUTURE PLAN}

Our investigation suggests that both the undergraduate and graduate students have many common difficulties with the fine structure corrections to the energy spectrum of a hydrogen atom using DPT. We used the difficulties found in this context as resources to develop a QuILT to help students develop a solid grasp of these concepts. The initial evalution of the QuILT is encouraging.

\section{ACKNOWLEDGEMENTS}

We are very grateful to the National Science Foundation for award PHY-1505460. We also thank all of the students and faculty members who helped with this investigation.
[1] M. Wittmann, R. Steinberg, and E. Redish, Am. J. Phys. 70, 218 (2002).

[2] D. Zollman, N. Rebello, K. Hogg, Am. J. Phys. 70, 252 (2002).

[3] C. Singh, Am. J. Phys. 69, 885 (2001).

[4] C. Singh, Am. J. Phys. 76, 277 (2008).

[5] C. Singh, Am. J. Phys. 76, 400 (2008).

[6] A. J. Mason and C. Singh, Am. J. Phys. 78, 760 (2010).

[7] S. Y. Lin and C. Singh, Euro. J. Phys. 31, 57 (2010).

[8] G. Zhu and C. Singh, Am. J. Phys. 80, 252 (2012).

[9] G. Zhu and C. Singh, Am. J. Phys. 79, 499 (2011).
[10] G. Zhu and C. Singh, Phys. Rev. ST PER 8, 010117 (2012).

[11] G. Zhu and C. Singh, Phys. Rev. ST PER 8, 010118 (2012).

[12] G. Zhu and C. Singh, Phys. Rev. ST PER 9, 010101 (2013).

[13] C. Singh, E. Marshman, Phys. Rev. ST PER 11, 020117 (2015).

[14] E. Marshman and C. Singh, Euro. J. Phys. 37, 024001 (2016).

[15] E. Marshman and C. Singh, Euro. J. Phys. 38, 045701 (2017).

[16] E. Marshman and C. Singh, Euro. J. Phys. 38, 025705 (2017).

[17] E. Marshman and C. Singh, Euro. J. Phys. 39, 015707 (2018).

[18] S. Siddiqui and C. Singh, Eur. J. Phys. 38, 035703 (2017).

[19] D. Griffiths, Prentice Hall, Upper Saddle River, NJ (2005). 